\title{
El modelo geoidal regional GGM-CA-2015, resultados y experiencia de la cooperación técnica en la región México, Centroamérica y el Caribe
}

\author{
David Ávalos-Naranjo* \\ Raúl Muñoz-Abundes* \\ Christopher Ballesteros ${ }^{+}$ \\ Wilmer Medrano-Silva ${ }^{\triangle}$ \\ Álvaro Álvarez-Calderón ${ }^{\square}$ \\ Carlos Figueroa $^{\circ}$ \\ Vinicio Robles-Pereira ${ }^{\boldsymbol{}}$ \\ Oscar Meza \\ Leopoldo Taveras $\bullet$
}

Recibido el $1^{\circ}$ de junio de 2016; aceptado el 24 de septiembre de 2016

\begin{abstract}
On recent times the federal agencies in charge of administrating the basic geographic information have received from the scientific community a clear recommendation to migrate the way vertical control coordinates are determined, moving from the local reference of mean sea level to a reference in terms of potential of the gravity field, like the geoid. The main advantage of such change is the ability to make the vertical datum compatible to that of any other country in the world and with the GNSS technology for surveying. In preparation for substituting the classic system,

* Dirección General de Geografía y Medio Ambiente, Instituto Nacional de Estadística y Geografía (INEGI), Av. Héroe de Nacozari Sur 2301, Fraccionamiento Jardines del Parque, C.P. 20276, Aguascalientes, México, correo electrónico: david.avalos@inegi.org.mx

+ Instituto Geográfico Nacional Tommy Guardia _-IGNTG, Panamá, pherballe@hotmail.com

$\triangle \quad$ Instituto Nacional de Estudios del Territorio _ INETER, Nicaragua, medranoswil@yahoo.com

- Instituto Geográfico Nacional__IGNCR, Costa Rica, aalvarez.igncr@gmail.com

○ Centro Nacional de Registros _-IGN/CNR, El Salvador, cfigueroa356@yahoo.com

- Instituto Geográfico Nacional__IGN, Guatemala, viniciorp@yahoo.com

- Instituto de la Propiedad -IP, Honduras, omeza1257@hotmail.com

- Instituto Cartográfico Militar_-ICM, República Dominicana, leotaveras@yahoo.es
\end{abstract}


based on observations to the local sea level, a large amount of geographic agencies face the challenge of implementing the new concepts with new methodology and data. In this work we introduce the first results from an effort in international collaboration from 8 countries in the region of Mexico, Central America and the Caribbean to increase the technical capability in this subject and diminish the breach between the scientific advancement and the practical implementation for the geodetic data infrastructure in official use.

The gravimetric geoid for Mexico, Central America and the Caribbean, GGMCA-2015 is introduced as an original digital model in coverage and resolution. It represents an equipotential surface of the gravity field, parameterized by the constant value $W_{0}=62636856.0 \mathrm{~m}^{2} / \mathrm{s}^{2}$. This realization allows to refer vertical coordinates for geo-positioning among a large amount of countries and islands on a consistent datum through national borders and across the oceans.

The processing of the GGM-CA-2015 was carried out by the Stokes-Helmert technique applied on the current coverage of gravimetric databases and takes advantage of the satellite information contained in the model GOCO05s. It is estimated that the accuracy of the geoidal heights obtained is on the level of decimeters, varying for zones according to the available observations of GNSS on benchmarks.

Additional to this, the document contains a discussion on a few identified ways to improve the geoid modeling in the region, regarded as a note for future work. At the same time it is underlined the advantage of the strategy implemented for a coordinated work which was chosen among representatives of the geographic agencies involved.

Key words: geoid modelling, Central America and Caribbean, gravity data, Stokes-Helmert.

\section{Resumo}

Em épocas recentes as agências federais encarregadas de administrar informação geográfica básica têm recebido por parte da comunidade científica uma clara recomendação de mudança sobre a forma de se determinar o controle de coordenadas verticais, migrando de referência local de nível médio do mar até uma referência definida em termos de potencial do campo de gravidade como o geoide. A principal vantagem desta mudança é a habilidade de fazer compatível o datum vertical entre países de qualquer parte do mundo e compatível com a tecnologia de levantamentos GNSS. Em preparação para substituir eventualmente o sistema clássico, baseado em observações ao nível do mar local, uma grande quantidade de agências enfrentam o desafio de implementar o conceito mencionado, com dados e metodologia novos. Neste trabalho apresentamos os primeiros resultados de um esforço de colaboração internacional surgido em 8 países na região do México, América Central e Caribe para incrementar a capacidade técnica nesta matéria e diminuir a brecha existente 
entre o avanço científico e a implementação prática de infraestrutura de dados geodésicos para uso oficial.

O geoide gravimétrico para o México, América Central e Caribe, GGM-CA2015, se apresenta como um modelo digital inédito em cobertura e resolução. Representa uma superfície equipotencial do campo de gravidade, parametrizado pela constante $W_{0}=62636856.0 \mathrm{~m}^{2} / \mathrm{s}^{2}$. Esta realização permite referir as coordenadas verticais para geolocalização de uma grande quantidade de países e ilhas sobre um datum consistente através de fronteiras e mares.

O processamento do GGM-CA-2015 se levou a cabo segundo a técnica StokesHelmert, com bases de dados gravimétricos atualizados e tomando vantagem do campo de gravidade modelado com informação satelital GOCO05s. Se estima que o grau de exatitude alcançado para as alturas geoidais é variável por zonas e segundo a informação disponível de observações GNSS sobre bancos de nível se obteve um desvio padrão de alguns decímetros.

No documento se discutem algumas vias identificadas para melhorar o modelado geoidal da região como aportes para trabalho futuro. Ao mesmo tempo se ressaltam vantagens da estratégia de trabalho coordenado que se adotou a seguir entre as agências geográficas envolvidas.

Palavras chave: modelado geoidal, América Central e Caribe, dados de gravidade, Stokes-Helmert.

\section{Resumen}

En épocas recientes, las agencias federales encargadas de administrar información geográfica básica, han recibido por parte de la comunidad científica una clara recomendación de cambio sobre la forma de determinar el control de coordenadas verticales, migrando de la referencia local de nivel medio del mar hacia una referencia definida en términos de potencial del campo de gravedad como es el geoide. La principal ventaja de este cambio radica en la habilidad de hacer compatible el dátum vertical entre países de cualquier parte del mundo y compatible con la tecnología de levantamientos GNSS. En preparación para sustituir eventualmente al sistema clásico, basado en observaciones al nivel del mar local, una gran cantidad de agencias enfrentan el reto de implementar el concepto mencionado, con datos y metodología nuevos. En este trabajo presentamos los primeros resultados de un esfuerzo de colaboración internacional surgido desde ocho países en la región de México, Centroamérica y el Caribe para incrementar la capacidad técnica en esta materia y disminuir la brecha existente entre el avance científico y la implementación práctica de infraestructura de datos geodésicos para uso oficial.

El geoide gravimétrico para México, Centroamérica y el Caribe GGM-CA-2015 se presenta como un modelo digital inédito en cobertura y resolución. Representa una superficie equipotencial del campo de gravedad, parametrizado por la constante 
$W_{0}=62636856.0 \mathrm{~m}^{2} / \mathrm{s}^{2}$. Esta realización permite referir las coordenadas verticales para geolocalización de una gran cantidad de países e islas sobre un dátum consistente a través de fronteras y mares.

El procesamiento del GGM-CA-2015 se llevó a cabo bajo la técnica StokesHelmert, con bases de datos gravimétricas actualizadas y tomando ventaja del campo de gravedad modelado con información satelital GOCO05s. Se estima que el grado de exactitud logrado para las alturas geoidales es variable por zonas y según la información disponible de observaciones GNSS sobre bancos de nivel se obtuvo una desviación estándar de algunos decímetros.

En el documento se discuten algunas vías identificadas para mejorar el modelado geoidal de la región como apuntes para trabajo futuro. Al mismo tiempo se resaltan ventajas de la estrategia de trabajo coordinado que se adoptó a seguir entre las agencias geográficas involucradas.

Palabras clave: modelado geoidal, Centroamérica y Caribe, datos de gravedad, Stokes-Helmert.

\section{Introducción}

Entre las políticas de manejo de la infraestructura de datos espaciales por lo general se promueve avanzar hacia la adopción de nuevos estándares internacionales que en ocasiones son de difícil implementación porque implican una inversión extraordinaria en recursos materiales o humanos. Tal es el caso de algunas capas de información geográfica básica como la información geodésica para el control de coordenadas verticales, sobre la cual se pretende edificar sistemas de geolocalización más modernos y complejos, compatibles a nivel internacional. Precisamente a nivel mundial en Julio de 2016 el comité experto de Naciones Unidas sobre Manejo de Información Geoespacial Global (UN-GGIM) publicó un plan estratégico en donde se recomienda el desarrollo de sistemas de alturas nacionales en coordinación con la Asociación Internacional de Geodesia (AIG) (UN-GGIM, 2016). Al mismo tiempo, al seno de la AIG se ha promovido la evolución de los sistemas de alturas hacia la adopción de un geoide como superficie de referencia para los sistemas de alturas. No obstante, el mismo documento de UN-GGIM advierte en muchos países que hay una falta de infraestructura necesaria para desarrollar tales sistemas.

En este reporte mostramos el resultado concreto de un esfuerzo orientado en los términos que marcan las tendencias y recomendación de la Organización de las Naciones Unidas (ONU), respecto al Marco Geodésico de Referencia Mundial para el Desarrollo Sostenible (GGRF). La filosofía de este trabajo atiende a principios como la equidad, la compartición de datos, cooperación multilateral y divulgación de resultados. Comenzamos con una descripción conceptual del geoide y su uso como componente del marco geodésico de referencia. 
Hoy en día, la mayor parte de la información geográfica generada en cada país está íntimamente ligada a los Sistemas de Navegación Global por satélites (GNSS, Global Navigation Satellite System). En la práctica es cada vez más difícil encontrar ejemplos de geolocalización realizados con alguna técnica distinta. Las aplicaciones concretas de geolocalización 3D que se basan en GNSS se benefician de contar con un estándar de referencia para expresar los datos de elevación sobre el nivel del mar. Un ejemplo son los levantamientos terrestres, aéreos y satelitales con los que se confecciona un modelo digital del terreno que es necesario para producir simulaciones en escenarios de riesgo por inundación. En estos casos una buena precisión en los valores de altura del modelo es clave para lograr un pronóstico de inundaciones realista, ya que las fallas en esta precisión derivan en escenarios muy distintos. Puesto que los modelos provienen de observaciones GNSS (e.g. levantamientos GPS sobre terreno, LiDAR o radar satelital) es un hecho que sus valores de elevación están determinados como alturas geodésicas o elipsoidales $(h)$. Para transformarlos en alturas ortométricas $(H)$, que son apropiadas para describir fenómenos físicos como el nivel del agua, es imprescindible contar con un modelo geoidal.

El modelo geoidal describe la separación vertical variable $(N)$ que existe desde el cero de altura en coordenadas geodésicas (el elipsoide) hasta el cero o dátum de las alturas ortométricas (el geoide). Para utilizar al modelo geoidal como herramienta de transformación se aplica la Ecuación 1, que gráficamente se explica en la Figura 1.

$$
H=h-N
$$

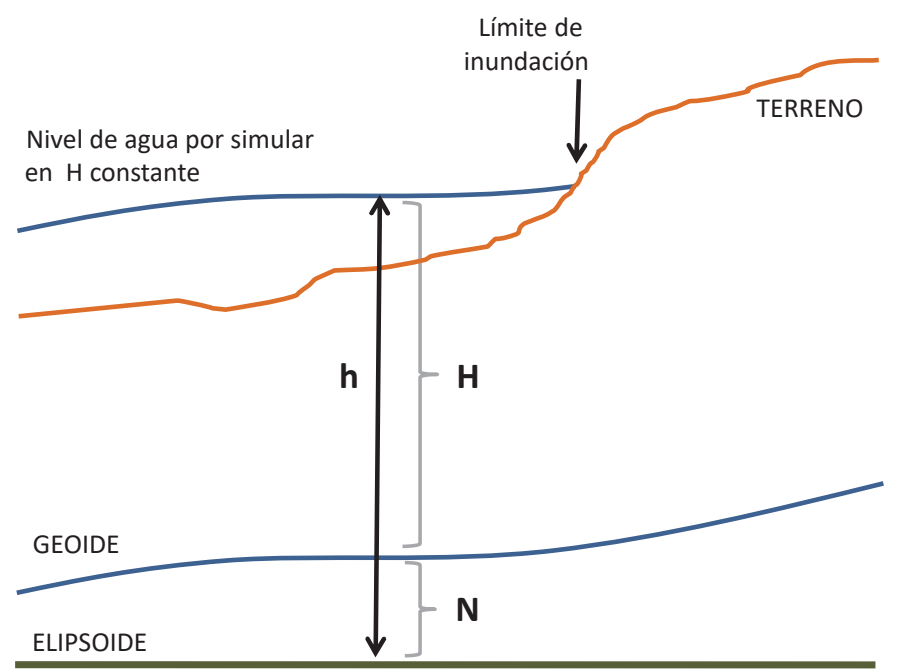

Figura 1. Esquema de relación entre alturas geodésicas y ortométricas. 


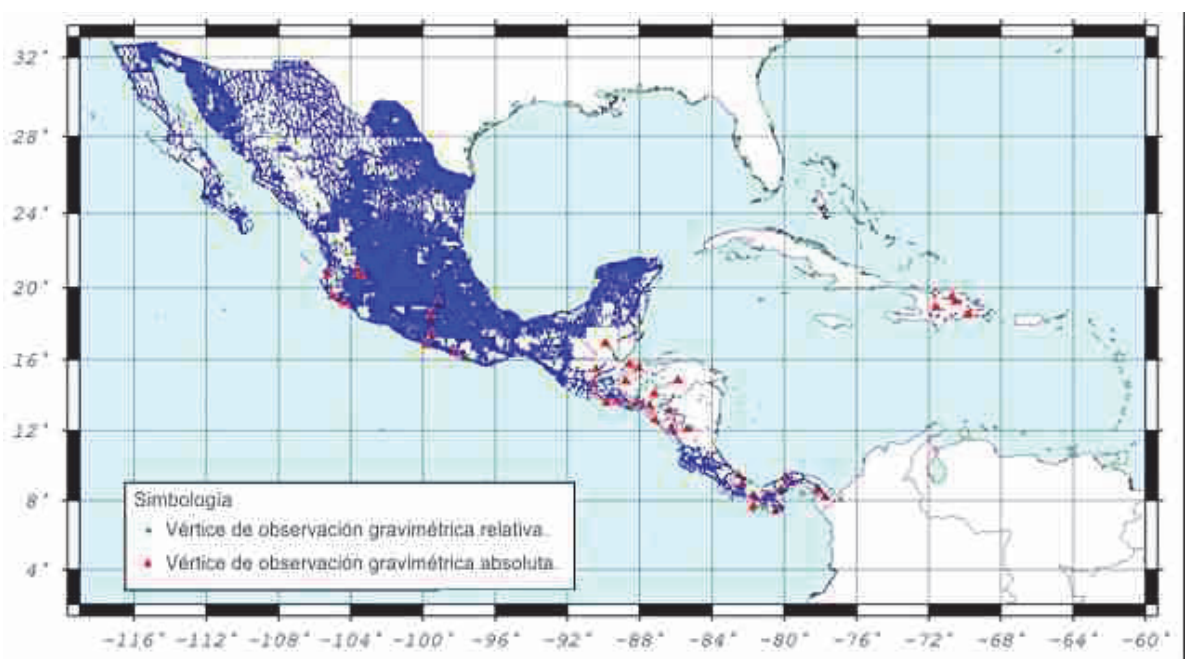

Figura 2. Distribución espacial de datos gravimétricos terrestres colectados o producidos directamente por parte de los institutos geográficos participantes.

Los avances científicos existentes ya dan soporte teórico para la obtención de modelos geoidales con precisión de pocos centímetros e incluso milímetros de error en los valores de $N$. Sin embargo, en la realidad solo algunos países logran aplicar esos avances de forma autónoma. Para la mayoría es preferible generar alianzas, fungiendo como productor de los datos más básicos para que su contraparte genere el análisis especializado que involucra un cálculo de alturas geoidales. El problema que esta situación genera es un apartamiento progresivo entre la capacidad técnica de la agencia y la vanguardia científica, en ocasiones nombrado brecha tecnológica.

Dos asuntos principales se documentan en este artículo, siendo el primero de ellos una revisión al esquema de colaboración efectuado para disminuir dicho apartamiento. El segundo asunto es una descripción del producto obtenido en 2015: el modelo geoidal regional nombrado GGM-CA-2015, el cual fue creado a partir de datos básicos de aceleración de la gravedad levantados en campo y aplicando una técnica de procesamiento con efectividad documentada.

Entre los resultados de esta experiencia se logró alcanzar una serie de objetivos como los siguientes:

a) Se consolidó una red de especialistas en manejo de información gravimétrica para ocho países de la región, 
b) Se recuperó información antigua y se integró un archivo unificado de datos gravimétricos,

c) Se puso en práctica un método novedoso de detección de errores gruesos en datos gravimétricos,

d) Se adquirió práctica en el manejo de software científico para cálculo geoidal,

e) Se produjo un nuevo mapa regionalizado del campo de gravedad,

f) Se produjo el modelo geoidal GGM-CA-2015

Se aclara que en este estudio mostramos la forma en que fueron solucionados algunos aspectos prácticos para lograr implementar la técnica de cálculo seleccionada, mientras que la búsqueda de desarrollos innovadores para mejorar la técnica queda fuera del objetivo que aquí se persigue. Consideramos que los elementos aportados demuestran la factibilidad de aplicar un esquema de trabajo coordinado y dirigido por políticas de operación consensadas, tanto para obtener el modelo geoidal de otras regiones, como para alcanzar distintos objetivos de cooperación técnica.

Las secciones siguientes muestran en forma sistemática los pasos de procesamiento, desde la procedencia y tratamiento de datos básicos hasta una estimación de la calidad del GGM-CA-2015.

\section{Recolección de datos}

Valores de aceleración de la gravedad levantados en campo y un modelo digital de elevación de cubrimiento continuo son los conjuntos de datos primarios. Durante un primer taller de capacitación se abordó el estudio de los levantamientos de gravimetría preexistentes en ocho países: República Dominicana, Panamá, Costa Rica, Nicaragua, Honduras, El Salvador, Guatemala y México. De manera consensada se determinó un formato de intercambio y se integró el conjunto de datos para distribuirse entre los participantes. Con esto se abrió la primera ganancia equitativa.

En un análisis exploratorio se verificó que la cantidad de información gravimétrica disponible es insuficiente en cantidad y en cubrimiento de los ocho países para efectuar un cálculo geoidal con precisión centimétrica. A pesar de que existen levantamientos recientes, en los que se estima que este esfuerzo colectó cerca de 35,000 datos gravimétricos más que los utilizados en la creación del modelo EGM2008, aún se detectan zonas extensas con carencia de un cubrimiento apropiado. Los nuevos datos se distribuyen principalmente en México, El Salvador y Panamá. En el presente trabajo se estima que la densidad mínima de datos gravimétricos necesarios para el cálculo geoidal de resolución 2.5' es de 1 punto por cada celda en zonas planas y de 12 puntos en zonas montañosas (Janak y Vanicek, 2011). 
La desventaja de cobertura en datos de entrada implica que la calidad de los modelos geoidales se vea disminuida con respecto a lo que el soporte teórico es capaz de generar. Reportes anteriores de modelos geoidales ya habían informado de esta desventaja y refieren, ya sea por cifras o por mapas de incertidumbre, que para la región de México, Centroamérica y el Caribe la confiabilidad del modelo es relativamente mala (e.g. Pavlis et al., 2012; Blitzkow et al., 2016).

\section{Gravimetría terrestre}

El método de levantamiento con gravímetros relativos es actualmente el único que los países de la región implementan para incrementar sus bases de datos. Las estaciones de referencia con valor de gravedad previamente conocido corresponden mayormente en algún ajuste como el IGSN71 y RELANG77; solo en pocos puntos se tomaron sitios de observación con gravímetros absolutos moderno con precisión por debajo de los 10 microGal. Debe tomarse en cuenta que estos levantamientos pueden tomar como referencia diferentes dátum de un país a otro (Avalos et al., 2013). Para la creación del modelo GGM-CA-2015 se utilizó la información de gravimetría relativa que se muestra en la Figura 2 y en la Tabla 1. El total de los valores de gravedad utilizados cuentan con la corrección de marea proporcionada por cada país participante.

Tabla 1

Cantidad de valores puntuales gravimétricos por país

\begin{tabular}{lr}
\hline \multicolumn{1}{c}{ País } & Puntos \\
\hline México & 80,744 \\
El Salvador & 339 \\
República Dominicana & 42 \\
Costa Rica & 2,312 \\
Nicaragua & 429 \\
Panamá & 1,195 \\
Guatemala & 1,371 \\
Honduras & 38 \\
\hline
\end{tabular}

La cobertura necesaria de puntos de gravimetría para el cálculo del modelo de alturas geoidales abarca desde los Estados Unidos de América hasta la zona norte de América del Sur. Para cubrir con información el área completa, a partir de $20 \mathrm{~km}$ desde cualquier punto con gravimetría terrestre, se tomó la gravimetría premodelada del modelo global EIGEN-6c3stat, completo a grado y orden 2190 el cual incorpora bases de datos más extensas e incluye un procesamiento especial para disminuir la presencia de sesgos locales. La Figura 3 muestra la cobertura correspondiente a este insumo. 


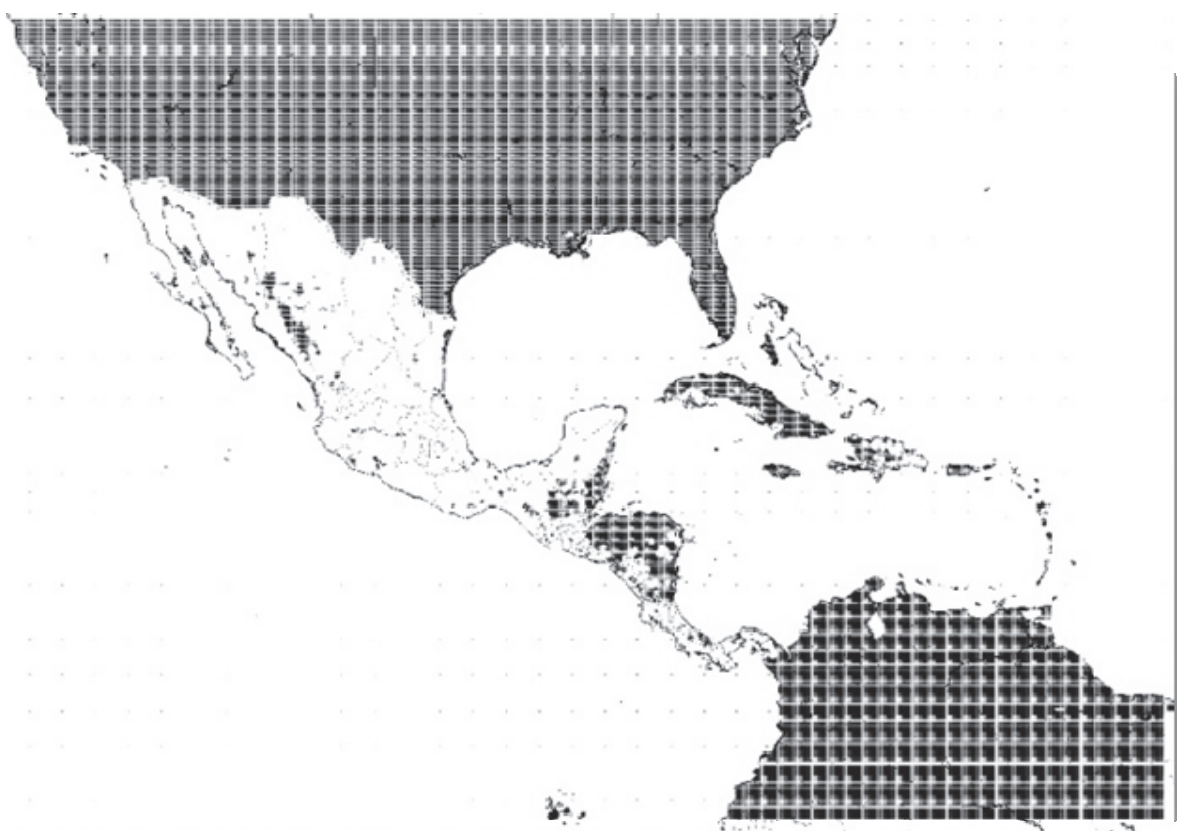

Figura 3. Distribución de los datos gravimétricos tomados del modelo EIGEN-6c3stat.

\section{Gravimetría marina}

El cubrimiento marino de información gravimétrica fue tomado del modelo DTU10, que proviene del procesamiento de mediciones satelitales sobre el nivel del mar por más de 10 años de observación de las misiones TOPEX/POSEIDÓN y Jason-1/2.

\section{Altura geoidal por GPS sobre bancos de nivel}

Clásicamente los modelos geoidales suelen ser comparados con mediciones terrestres de altura geodésica mediante técnicas GNSS, combinadas con valores de altura del terreno sobre bancos de nivel. Usando la fórmula $\mathrm{N}=\mathrm{h}-\mathrm{H}$, se obtuvo el valor de altura geoidal para un conjunto de 301 puntos distribuidos en 4 países.

\section{Modelos digitales del terreno}

Los modelos digitales de terreno son indispensables para efectuar estimaciones del efecto de la topografía sobre el campo de gravedad. Por características de disponibilidad, alta resolución y cubrimiento homogéneo en la región completa, se eligió trabajar con el modelo digital de elevaciones SRTM3 (Shuttle Radar Topography 
Mission). La resolución original descargada corresponde a 3" (aproximadamente 90 metros), suficiente para reproducir con fidelidad los efectos de la topografía en el campo de gravedad con resolución objetivo de 2.5'. Adicionalmente se utilizó el modelo de alturas medias SRTM30 y uno más calculado en resolución de 2.5', ambos con cobertura de hasta $10^{\circ}$ alrededor del área objetivo del modelo geoidal. Para cubrimiento mundial completo, se colectó también el modelo JGP95 en resolución de $1^{\circ}$. Estos formatos permiten al software de cálculo geoidal ser eficiente en la estimación de los efectos de masas topográficas globales.

La adquisición y manejo de modelos digitales se produjo distribuyendo la carga de trabajo entre los participantes, integrando luego las partes y compartiendo la colección completa de modelos.

\section{Modelado del campo de gravedad regional}

El primer análisis de datos se consolidó en un modelo digital de anomalías de gravedad de aire libre sobre el terreno. En esta sección se describe el tratamiento de información implementado en dos etapas: limpieza de datos e interpolación.

\section{Limpieza de datos}

Con motivo de homogeneizar la calidad y dátum del valor de altura asignado a los valores de gravedad colectados, se calcularon los valores de anomalía de gravedad $(\Delta g)$ respectivos utilizando solo una fuente del valor de altura: el modelo SRTM3. De este modo se obtuvo también compatibilidad con cálculos posteriores de efectos topográficos basados en el mismo modelo de elevaciones.

Se aplicó un control de calidad a los datos de gravedad que consistió en calcular y mapear los valores puntuales de anomalía de Bouguer completa en aproximación esférica $\left(\Delta g^{B C}\right)$. De acuerdo al planteamiento de Janak y Vanicek, 2004, el campo de $\Delta g^{B C}$ es menos rugoso que el de $\Delta g$, entonces la presencia de puntos con valor discrepante se hace más evidente. Los valores puntuales pueden graficarse de diversas maneras en mapas de distribución espacial; sin embargo en sustitución de los estilos clásicos de mapeo se tomó ventaja del software de visualización de datos geográficos llamado Google-Earth. El software de acceso en línea se muestra aquí como una herramienta útil en la identificación de datos erróneos. Para ello se creó una serie de archivos con cubrimiento parcial de la región de estudio, donde los valores $\Delta g^{B C}$ escalados por 10 se disponen a manera de altura sobre el terreno. La representación gráfica que esto produce en Google Earth se presenta en la Figura 4, donde puede notarse que de manera visual es factible identificar puntos fuera de un patrón de comportamiento local. 


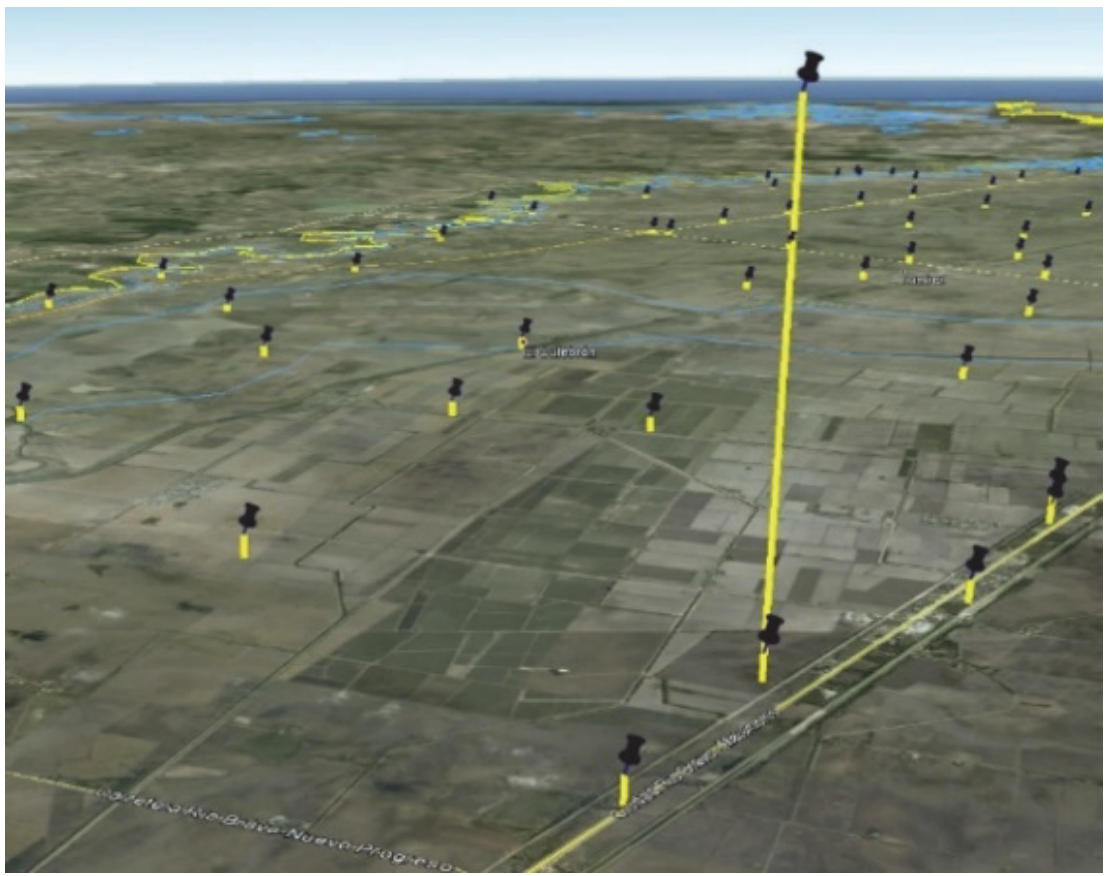

Figura 4. Un ejemplo del método empleado para detectar datos gravimétricos inconsistentes. La línea vertical que sobresale de las demás corresponde a un dato gravimétrico inconsistente con su entorno.

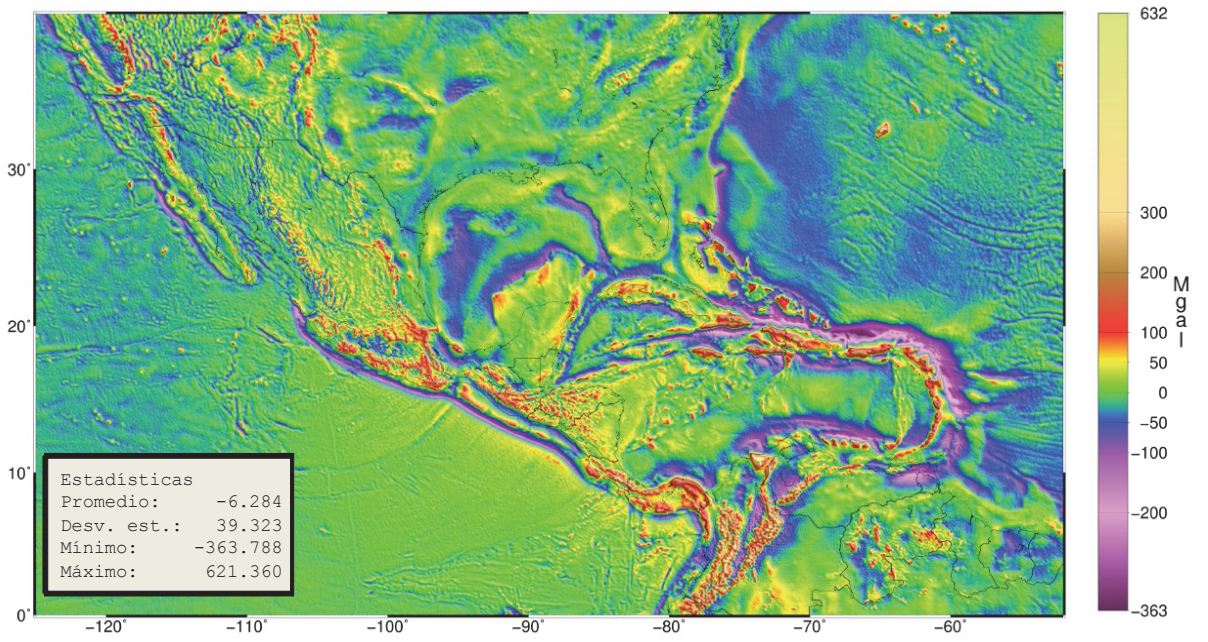

Figura 5. Mapa general de anomalías de aire libre sobre el terreno modeladas. 


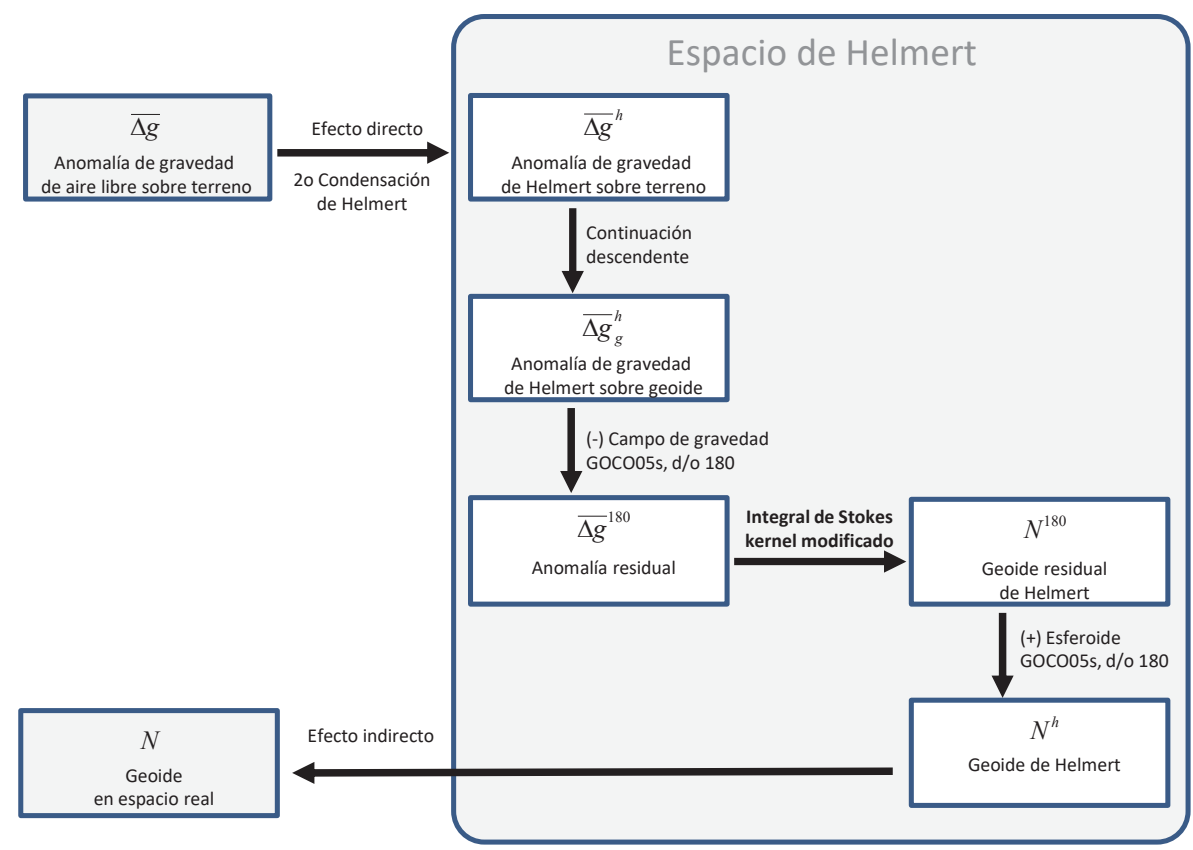

Figura 6. Diagrama de las etapas de cálculo geoidal aplicadas con la técnica StokesHelmert.

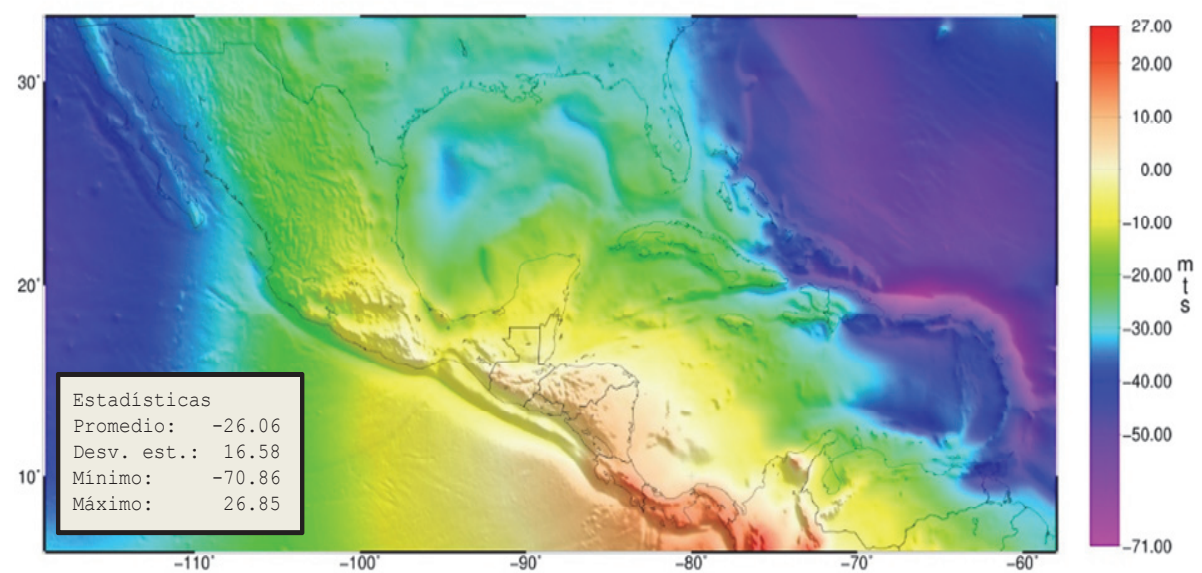

Figura 7. Mapa de alturas geoidales del modelo GGM-CA-2015. 
Tras un ejercicio de exploración visual exhaustiva por toda la cobertura se identificó un total de 536 puntos discrepantes, a los cuales se les separó del grupo de datos de entrada por contener algún error relacionado con sus coordenadas o el valor de gravedad capturado. Esta selección de puntos fue basada en el criterio de personal experimentado en levantamientos gravimétricos, quienes sugieren tomar tiempo adicional para determinar si es posible corregir el error de cada caso. Las posibilidades de corrección dependen de la disponibilidad de información adicional que deberá revisarse en libretas de campo.

\section{Interpolación}

El conjunto de datos $\Delta g^{B C}$ validados se integró con los puntos de las Figuras 2 y 3 más los datos de cobertura marina. Luego se aplicó un proceso de interpolación en dos etapas. Primeramente, se creó un modelo temporal de resolución relativamente alta (15 segundos de arco) por el método de interpolación bicúbica. Con ello se obtuvo una cantidad de datos significativa al interior de cada celda de resolución $2.5^{\prime}$ x $2.5^{\prime}$ para estimar un promedio. En segundo lugar se efectuó un filtro de promediado simple que arrojó un solo valor representativo de cada celda en cubrimiento de latitud Norte $0^{\circ}$ a $39^{\circ}$ y de longitud Este $235^{\circ}$ a $308^{\circ}$. El valor promedio de la anomalía de Bouguer obtenido de esta forma $\left(\overline{\Delta g^{B C}}\right)$ es representativo.

A partir del modelo digital $\overline{\Delta g^{B C}}$ se calculó el valor medio de anomalías de aire libre sobre el terreno $(\overline{\Delta g})$ con la misma resolución usando la Ecuación 2.

$$
\overline{\Delta g}=\overline{\Delta g^{B T}}+\overline{C T}-2 \pi G \rho \bar{H}
$$

donde $\overline{C T}$ representa la corrección de terreno promedio calculada en aproximación esférica y $\bar{H}$ es la elevación media del terreno en resolución de 2.5', derivada del modelo SRTM3. El valor de corrección de terreno $\overline{C T}$ en cada celda corresponde al promedio de 100 puntos de cálculo ubicados equidistantemente al interior de la celda. Las elevaciones fueron tomadas del modelo SRTM3 hasta un radio de $3^{\circ}$ alrededor de cada punto de cálculo y la densidad topográfica asignada fue la constante de $\rho=2670 \mathrm{~kg} / \mathrm{m}^{2}$. En resumen el flujo de datos puede representarse con la siguiente secuencia de términos (3) y el mapa resultante se presenta en la Figura 5.

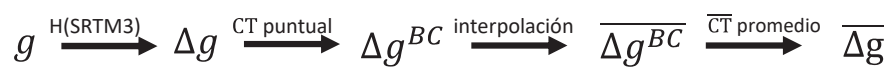

Este esquema de procesamiento es benéfico para mantener reducida la magnitud de sesgos al interpolar sobre zonas de escasa información (Janak y Vanicek, 2004). 
No obstante se reconoce que existen algunas técnicas de procesamiento recientemente liberadas que tienen el potencial de mejorar este trabajo, como es la estimación de topografía residual para detallar aún más el campo de gravedad en zonas costeras y lacustres (Hirt, 2013), con las que podría generarse un refinamiento de exactitud para $\overline{\Delta g}$. Es importante notar que este tipo de procesamiento más complejo quedó fuera del plan de trabajo trazado inicialmente (INEGI, 2011). Consideramos que las estrategias de mejora como esta deben ser tomadas en cuenta como trabajo futuro.

En cuanto a la coordinación de actividades de esta etapa cabe resaltar que se implementó un procesamiento en paralelo dividiendo cargas de trabajo por zonas geográficas. Con ello se logró que los participantes mantuvieran control de todos los pasos del modelado. El mosaico de resultados fue integrado en México y devuelto a los participantes con el fin de brindar la oportunidad de revisar y validar la pertinencia del nuevo modelo.

\section{Parametrización para el modelado}

Actualmente los países de la región México, Centroamérica y el Caribe cuentan con estándares nacionales de superficie de altura cero desligados entre sí (e.g. Avalos et al., 2013). No obstante, los participantes de este trabajo ya alcanzaron un acuerdo sobre el parámetro estándar que define el nivel de elevación del GGM-CA-2015. Este parámetro es el valor de potencial de gravedad constante $W_{0}=62636856.0 \mathrm{~m}^{2} / \mathrm{s}^{2}$, tomado del IERS entre su lista de parámetros globales asociados al elipsoide GRS80. Durante el 3er. Taller del Geoide en Aguascalientes, México, en septiembre de 2014, se discutió a nivel técnico sobre una serie de valores $W_{0}$, todos distintos y propuestos como recomendación desde grupos de trabajo independientes. Incluso se consideró la posibilidad de calcular y proponer otro valor, adicional a los ya publicados. Finalmente se decidió (siete votos contra uno) privilegiar la parametrización del IERS puesto que los marcos de referencia nacionales refieren al GRS80 como su elipsoide de referencia según la definición del propio IERS.

Sin ninguna pérdida de generalidad, el GGM-CA-2015 se creó adoptando el parámetro mencionado. Considerando que solo existen centímetros o pocos decímetros entre éste y otros valores $W_{0}$, mientras que la diferencia real entre niveles medios del mar de la región es ciertamente de mayor magnitud. Es decir, que cuando se selecciona un nivel $W_{0}$ para el geoide regional, entonces algunos países se pueden ver beneficiados porque dicho nivel es cercano al de su dátum oficial, mientras que otros se verían perjudicados porque para ellos se incrementa esa diferencia. Dado que ninguno de los valores $W_{0}$ puede producir un beneficio equitativo, entonces el apoyo a estándares previos en uso efectivo resulta ser una mejor opción. Esto último trae con sigo una ventaja real, que es la de evitar traer más parámetros 
de transformación, facilitando a todos los usuarios la combinación de datos entre regiones.

El mismo valor de referencia $W_{0}$ que se adoptó para el GGM-CA-2015 fue adoptado para referencia oficial en Estados Unidos y en Canadá. Por otro lado, en julio de 2015 la Asociación Internacional de Geodesia emitió una resolución acerca del valor $W_{0}$ mundial, para el cual hoy se promueve su uso. Para todos los efectos prácticos, la diferencia con el nuevo parámetro es de $2.5 \mathrm{~m}^{2} / \mathrm{s}^{2}$ (Sánchez et al., 2016), que equivale a $27 \mathrm{~cm}$ por encima del GGM-CA-2015. Esta distancia puede tomarse como una constante de transformación entre las alturas ortométricas determinadas con el GGM-CA-2015 y el reciente valor $W_{0}$ por ser realizado como estándar global.

A continuación se describen los conceptos y ecuaciones que dieron paso a la realización del modelo geoidal para la superficie de geopotencial constante $\mathrm{W}_{0}=62636856.0 \mathrm{~m}^{2} / \mathrm{s}^{2}$.

\section{Metodología de cálculo geoidal}

Cada valor de altura geoidal se obtuvo a través de la integral de Stokes generalizada, aplicada al campo de gravedad local y global, pero bajo condiciones especiales que permiten asegurar una mayor exactitud. Los conceptos teóricos implementados corresponden al esquema Stokes-Helmert (e.g. Ellmann y Vanicek, 2007; Vanicek et al., 2013). Esperamos que las secciones siguientes funcionen como un apoyo al lector para adquirir un panorama general de los pasos y criterios aplicados, mientras que al lector especializado se le invita a consultar las expresiones matemáticas completas en las referencias bibliográficas.

Primeramente, el campo de gravedad modelado que se utilizó de insumo en la integral corresponde a valores de anomalía de gravedad estimados al nivel del geoide en un espacio de Helmert $\left(\Delta g_{g}^{h}\right)$, en donde se cumple la condición de armonicidad para el espacio ubicado encima del geoide. Para obtener el modelo de estas características se aplicó un proceso en dos pasos, comúnmente conocidos como helmertización de las anomalías de aire libre y continuación descendente (véase Figura 6).

\section{Helmertización}

Dicho proceso consiste en sustituir el efecto de atracción gravitacional de las masas ubicadas encima del geoide (topografía y atmósfera) por el efecto de una capa delgada de materia colocada en el geoide, cuya densidad incorpora al equivalente de la masa original, pero compactada. El cálculo formal de este paso corresponde al segundo método de condensación de Helmert, 1884. La formulación de este paso se expresa en la siguiente ecuación: 


$$
\Delta g^{n}=\Delta g+D T E+D A E+S I T E+\chi+\varepsilon_{\delta g}-\varepsilon_{n}
$$

en donde $\Delta g$ es el valor de anomalía de gravedad de aire libre sobre la topografía estimada con mediciones sobre el terreno, DTE es el efecto topográfico directo, DAE es el efecto atmosférico directo y SITE es el efecto topográfico secundario indirecto. Todos estos efectos fueron calculados con las expresiones de Martinec et al., 1996. $\varepsilon_{\delta g}$ y $\varepsilon_{n}$ son términos de corrección elipsoidal que complementan el cálculo en aproximación esférica de los efectos mencionados (e.g. Vaníček et al., 2012). Una corrección más se considera con el símbolo $\chi$, que compensa la diferencia de anomalías entre el geoide y cuasigeoide. En este paso se usó el valor constante de densidad de masa topográfica $\rho=2670 \mathrm{~kg} / \mathrm{m}^{2}$, omitiendo los términos de corrección por variaciones de densidad. De acuerdo con Huang et al., 2001, este hecho induce un error de altura geoidal que podría alcanzar hasta $7 \mathrm{~cm}$ en ubicaciones de topografía con elevaciones mayores a 2,500 metros.

\section{Continuación descendente}

La relación entre el campo de gravedad referido al nivel de la topografía $\left(\Delta g^{h}\right)$ y el campo referido al nivel del geoide $\left(\Delta g_{g}^{h}\right)$ se planteó con la ecuación de Poisson (e.g. Heiskanen y Moritz, 1967, p. 317). Esta relación adquiere validez teórica en el espacio de Helmert y fue implementada en el campo de gravedad discreto con un sistema de ecuaciones lineales en forma matricial (Kingdon y Vanicek, 2011):

$$
\Delta g_{t}=B \cdot \Delta g_{g}
$$

donde la matríz $B$ contiene valores del kernel de Poisson calculados en promedio doble y el vector $\Delta g_{g}$ fue obtenido con un proceso de solución iterativo, siguiendo los mismos principios expuestos por Sun y Vanicek, 1998.

\section{Integración de Stokes}

La expresión generalizada para la integral de Stokes se utilizó para tomar ventaja de la separación espectral entre los componentes de frecuencia baja y los de frecuencia media-alta (Vanicek y Sjoberg, 1991). Para la frecuencia baja se tomó como esferoide de referencia el campo de anomalía de gravedad derivado de GOCO05s (Mayer-Gurr et al., 2015) hasta grado y orden 180. Ese nivel de resolución (aproximadamente de $220 \mathrm{~km}$ ) ha sido recomendado por varios autores para uso en procesos de precisión centimétrica. Usando un kernel modificado se condiciono la integral para procesar únicamente los componentes de ondulación geoidal de frecuencia media-alta, dando como resultado un modelo de geoide residual $\left(N^{M}\right)$ de acuerdo con la siguiente expresión: 


$$
N^{M}=\frac{R}{4 \pi \gamma_{0}} \oiint_{\Omega} S^{M}(\psi) \Delta g^{M} d \Omega
$$

donde $R$ es el radio medio de la Tierra, $\gamma_{0}$ es el valor de aceleración de gravedad sintética a nivel del geoide, $S^{M}$ es el kernel de Stokes modificado a grado $M=180$, $\psi$ es el ángulo geocéntrico entre el punto de cálculo y los datos modelados de gravedad, $\Omega$ es la posición sobre el geoide en términos de latitud y longitud $(\Omega=(\varphi, \lambda))$, y por último $\Delta g^{M}$ corresponde al campo de anomalías de gravedad residual a nivel del geoide. Este último se obtuvo a partir del modelo resultante de la continuación descendente y sustrayendo el campo de referencia de baja frecuencia $\left(\Delta g_{M}\right)$, derivado del modelo geopotencial GOCO05s para $M=180$; es decir:

$$
\Delta g^{180}=\Delta g_{g}^{h}-\Delta g_{180}
$$

Adicionalmente, para evitar la necesidad de integrar sobre un campo de gravedad mundial, se aplicó la integral en forma rigurosa hasta la distancia $\psi_{0}=6^{\circ}$. El resto de la contribución (llamado error de truncación) se calculó por separado (Vanicek y Sjoberg, 1991).

En concordancia con lo anterior, el modelo de altura geoidal en el espacio de Helmert $\left(N^{h}\right)$ se calculó agregando el geoide residual al esferoide de referencia helmertizado de grado 180 en sistema libre de marea:

$$
N^{h}=N_{180}+N^{180}
$$

Particularmente el término de grado uno fue despreciado $\left(N_{l}=0\right)$ y en el término de grado cero se consideró una corrección de escala $N_{0}=0.45 \mathrm{~m}$, para tomar en cuenta la diferencia media entre el elipsoide GRS80 y el nivel de potencial objetivo $W_{0}=62636856.0 \mathrm{~m}^{2} / \mathrm{s}^{2}$.

\section{Des-Helmertización}

Por último se aplicó un proceso de transformación de alturas geoidales al espacio real. Dado que al haber compactado el efecto de masas topográficas para llegar al espacio de Helmert esto genera una distorsión en el potencial de gravedad, el geoide calculado corresponde a este campo ligeramente deformado. Para esto se calculó el efecto topográfico indirecto primario (PITE) con la ecuación 9 que corresponde al tipo de condensación de Helmert empleado:

$$
N=N h+P I T E
$$


Ningún tipo de ajuste ni operación adicional se aplicó al modelo de altura geoidal. Con esta expresión se aclara que el tipo de solución producida para el GGM-CA-2015 es puramente gravimétrica.

\section{Resultados}

La puesta en práctica de los conceptos descritos arrojó una serie de modelos digitales que describen distintos aspectos del campo de gravedad. Para dar a conocer los resultados de esta serie completa de modelos se muestra a continuación el resumen estadístico de la Tabla 2 que ayuda como referencia general al lector especializado con interés de contrastar algunos cálculos producidos de forma independiente.

Tabla 2

Resumen estadístico de los valores de los modelos digitales involucrados en el procesamiento geoidal del GGM-CA-2015

\begin{tabular}{|c|c|c|c|c|c|c|}
\hline Modelo & Símbolo & Unidades & Media & $\begin{array}{c}\text { Desviación } \\
\text { estándar }\end{array}$ & Máximo & Mínimo \\
\hline Altura del terreno & $H$ & metros & 212.819 & 528.813 & 6074.000 & -83.000 \\
\hline $\begin{array}{l}\text { Anomalía de aire libre } \\
\text { sobre el terreno }\end{array}$ & $\Delta g$ & $\mathrm{mGal}$ & -6.284 & 39.323 & 621.360 & -363.788 \\
\hline $\begin{array}{l}\text { Anomalía de Bouguer } \\
\text { esférica }\end{array}$ & $\Delta g^{B C}$ & $\mathrm{mGal}$ & -10.166 & 35.315 & 441.484 & -363.555 \\
\hline $\begin{array}{l}\text { Corrección de terreno } \\
\text { esférica }\end{array}$ & $C T$ & $\mathrm{mGal}$ & 1.078 & 7.133 & 264.672 & -1.487 \\
\hline $\begin{array}{l}\text { Efecto topográfico } \\
\text { directo }\end{array}$ & $D T E$ & $\mathrm{mGal}$ & 0.035 & 3.751 & 61.061 & -150.192 \\
\hline $\begin{array}{l}\text { Efecto atmosférico } \\
\text { directo }\end{array}$ & $D A E$ & $\mathrm{mGal}$ & 0.848 & 0.046 & 0.874 & 0.470 \\
\hline $\begin{array}{l}\text { Efecto topográfico } \\
\text { indirecto secundario }\end{array}$ & SITE & $\mathrm{mGal}$ & -0.009 & 0.016 & -0.004 & -0.270 \\
\hline $\begin{array}{l}\text { Anomalia de Helmert } \\
\text { sobre la topografia }\end{array}$ & $\Delta g^{h}$ & $\mathrm{mGal}$ & -5.418 & 38.286 & 534.926 & -362.927 \\
\hline $\begin{array}{l}\text { Anomalia de Helmert } \\
\text { sobre el geoide }\end{array}$ & $\Delta g_{g}^{h}$ & $\mathrm{mGal}$ & -5.129 & 40.061 & 911.162 & -362.927 \\
\hline $\begin{array}{l}\text { Anomalía de Helmert } \\
\text { de referencia }\end{array}$ & $\Delta g_{180}$ & $\mathrm{mGal}$ & -5.948 & 30.545 & 233.467 & -297.371 \\
\hline Anomalía residual & $\Delta g^{180}$ & $\mathrm{mGal}$ & 0.673 & 26.506 & 796.314 & -381.386 \\
\hline $\begin{array}{l}\text { Geoide residual de } \\
\text { Helmert }\end{array}$ & $N^{180}$ & metros & -0.001 & 0.622 & 8.179 & -4.564 \\
\hline $\begin{array}{l}\text { Esferoide de } \\
\text { referencia }\end{array}$ & $N_{180}$ & metros & -25.601 & 16.563 & 25.296 & -68.951 \\
\hline Efecto topográfico & PITE & metros & -0.031 & 0.056 & -0.013 & -1.152 \\
\hline Indirecto primario & & & & & & \\
\hline Altura geoidal & $N$ & metros & -26.061 & 16.587 & 26.855 & -70.861 \\
\hline
\end{tabular}


En nuestra experiencia, la generación de estadísticos y mapas a cada etapa del proceso fue de gran utilidad como control de calidad, en donde se verificaron aspectos como: completitud de información, correspondencia con las características físicas del territorio, continuidad y consistencia interna entre los valores. Como muestra de entre las visualizaciones hechas para cada modelo, la Figura 7 presenta el Mapa de alturas geoidales del GGM-CA-2015 como la más significativa.

\section{Evaluación de exactitud del GGM-CA-2015}

Se tienen preparadas dos formas de estimar el nivel de exactitud general del modelo GGM-CA-2015. Primeramente, el método clásico de comparación contra datos puntuales GPS/BN, y en complemento se presenta una estimación por diferencias contra el modelo geopotencial EGM2008.

Presumiblemente la técnica GPS/BN puede llegar a ser de mayor precisión que un modelo geoidal gravimétrico; sin embargo esta técnica tiene también algunas limitantes que frecuentemente demeritan la confiabilidad de sus valores de altura geoidal derivada. Estas limitantes se refieren a metodologías de levantamiento GPS heterogéneas, inestabilidad vertical de las marcas y dátum nacionales discrepantes con efectos de distorsión poco estudiados. En total es posible que varios de los registros de altura geoidal puntual contengan errores decimétricos. Teniendo este hecho en mente, se presenta a continuación la estadística de diferencias de altura geoidal en términos absolutos.

La Tabla 3 muestra valores promedio de diferencia entre las alturas geoidales, que se interpretan como una primera estimación del parámetro de transformación por país entre el dátum nacional y el dátum $W_{0}$. La desviación estándar habla de la variación típica entre las fuentes comparadas y el número de puntos prescribe la representatividad de la muestra.

Tabla 3

Estadísticos de diferencia puntual entre la altura geoidal derivada de levantamientos GPS y el modelo GGM-CA-2015

\begin{tabular}{lccc}
\hline \multicolumn{1}{c}{ País } & $\begin{array}{c}\text { Promedio } \\
N_{G P S}-N_{G G M-C A} \\
(\mathrm{~m})\end{array}$ & $\begin{array}{c}\text { Desviación } \\
\text { estándar } \\
(\mathrm{m})\end{array}$ & $\begin{array}{c}\text { Núm. puntos } \\
\text { GPS } / \text { BN }\end{array}$ \\
\hline México & -0.29 & 0.22 & 65 \\
El Salvador & 0.14 & 0.36 & 51 \\
Guatemala & 0.36 & 1.03 & 73 \\
Nicaragua & 0.54 & 0.28 & 112 \\
\hline
\end{tabular}


La altura ortométrica de bancos de nivel está ligada a distintos dátum. Para El Salvador el dátum es La Unión 1960, en Guatemala es San José, en Nicaragua es Corinto 1952, y en México es NAVD88. La diferencia formal entre estos dátum no ha sido determinada. Los valores presentados en la columna de promedio de la Tabla 3 representan una aproximación al sesgo entre dátum nacionales. La magnitud de desviaciones estándar que se muestra a nivel de decímetros puede interpretarse como un indicador de la confiabilidad del modelo geoidal; sin embargo, se prevé que dichas cifras contienen una influencia no determinada de la distorsión propia de cada dátum nacional. Con motivo de mitigar la aparición de esta influencia se propone como trabajo futuro la investigación sobre medición de dichas distorsiones.

Una fuente independiente de modelado geoidal como el EGM2008 presenta una oportunidad de contrastar las alturas geoidales del modelo GGM-CA-2015. Se calculó el modelo geoidal a partir del EGM2008 con el potencial $W_{0}$ coincidente con el del GGM-CA-2015, agregando además el efecto de terreno sobre alturas geoidales provenientes de un modelo geopotencial según la definición de Huang y Véronneau, 2013. El mapa de diferencias resultante se presenta en la Figura 8, donde puede notarse que existen variaciones menores en zonas marítimas al igual que en zonas continentales de relieve bajo. No obstante, en múltiples zonas montañosas de tierra firme aparecen diferencias que pueden superar el medio metro y en otras zonas como América del Sur, Costa Rica, Guatemala y sur de México aparecen ondulaciones de magnitud aún mayor.

En el caso de El Salvador, aunque no se distingue algún salto tan prominente en la Figura 8, sí se presenta una diferencia cercana a 3 decímetros de tipo sistemático. Para este caso se estima que este efecto puede ser producido por un sesgo en los datos gravimétricos. En Honduras y Panamá las diferencias son moderadas y con longitud de onda similar a la de información satelital nueva. De modo que parece ser una influencia del modelo GOCO05s. Para Nicaragua se distingue una situación similar a la de Honduras, salvo por un par de sitios con tonalidad azul obscura que sugieren la posible existencia de datos gravimétricos exagerados. Los datos de esa zona son aislados y aparentemente discrepan de la información circundante que proviene del modelo EIGEN6c3stat. Los casos de República Dominicana, Costa Rica y México son similares en el sentido de que parecen estar influenciados por algún efecto topográfico adicional.

Una parte de las diferencias en la Figura 7 son de esperarse dado que el campo de gravedad fuente es distinto para ambos modelos geoidales. Esto se ilustra en la Figura 9, que contiene el efecto en altura geoidal producido precisamente por esas diferencias entre el campo de gravedad derivado de EGM2008 y el utilizado para construir el modelo GGM-CA-2015. Cada pieza del patrón que puede identificarse igual en ambas figuras significa que el nuevo modelo de anomalías de gravedad de 
aire libre está aportando cambios significativos respecto al EGM2008. Es el caso de áreas como el sur de México, Guatemala y Costa Rica.

En zonas marinas las diferencias más abultadas son producto del modelo DTU10, que representan una mejora respecto al modelo DTU08 utilizado en el EGM2008. En zonas continentales el modelo GGM-CA-2015 tiene la ventaja de contar con la información satelital GOCO05s, que es la más confiable de este tiempo, además de combinar cerca de 35,000 datos terrestres nuevos. De modo que las diferencias de resolución baja, correspondientes a longitud de onda de $2^{\circ}$ o más, pueden considerarse como una ventaja del nuevo modelo geoidal.

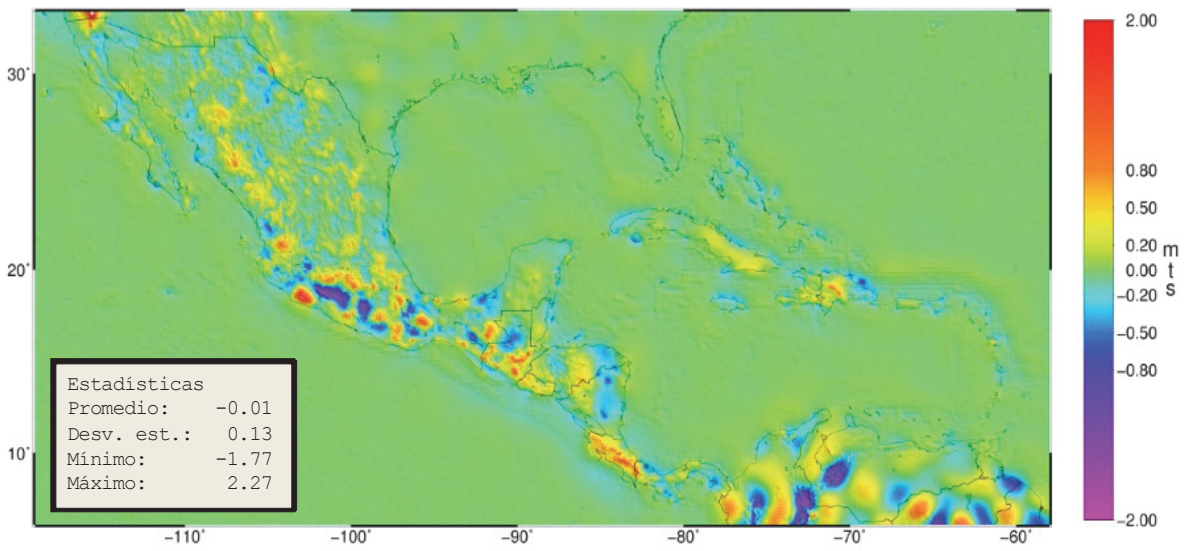

Figura 8. Diferencias de altura geoidal entre los modelos GGM-CA-2015- EGM2008.

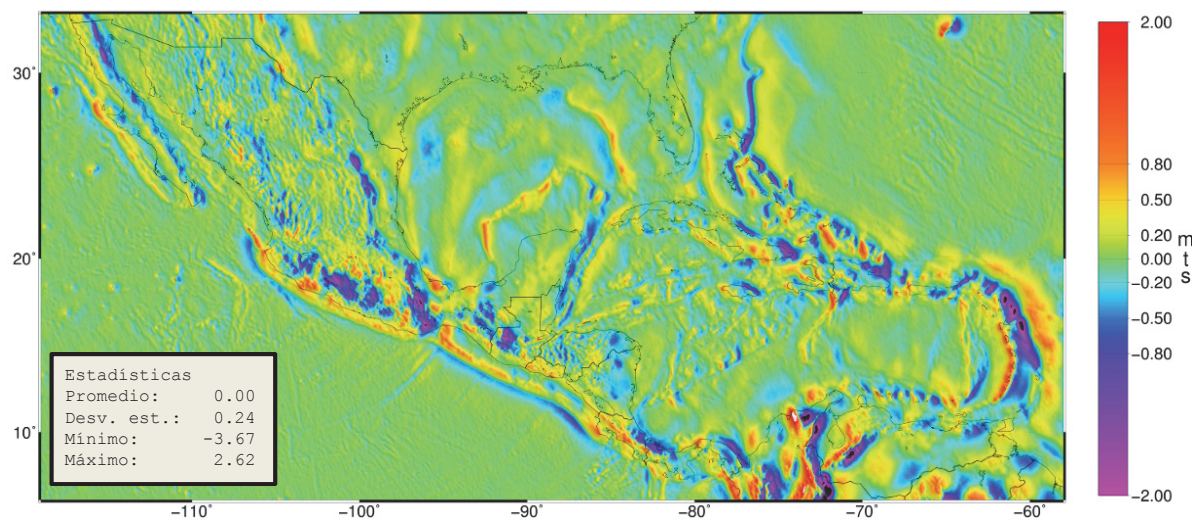

Figura 9. Diferencias de altura geoidal causadas por variación del campo de gravedad modelado. 
La Figura 9 se considera relevante porque ayudó a descubrir que en la Figura 8 existen diferencias adicionales que pueden alcanzar varios decímetros, aparentemente relacionadas con la altura del terreno. Aún queda por realizarse una investigación a fondo de las causas para estas diferencias adicionales porque debe descartarse la posibilidad de existencia de sesgos por algún error en el modelado de efectos topográficos o en los datos gravimétricos utilizados.

Se recomienda como trabajo futuro, para especialistas y para el propio grupo de trabajo del geoide, proponer técnicas de análisis enfocadas a validar la magnitud de efectos topográficos como DTE, SITE y PITE. También se recomienda producir estudios individuales por país que ayuden a determinar la precisión y exactitud de sus conjuntos de datos GPS/BN al igual que con los datos gravimétricos provenientes de levantamientos antiguos.

\section{Conclusiones}

La cooperación técnica entre instituciones de ocho países ha logrado liberar una primer serie de productos cartográficos inéditos con cobertura regional de México, Centroamérica y el Caribe. Se presentó el modelo digital de anomalía de aire libre sobre terreno y el modelo digital de alturas geoidales GGM-CA-2015, ambos creados con insumos actuales, tecnología probada y una alta resolución de 2.5 minutos de arco.

Se concentró una base de datos gravimétricos validada para uso común de las ocho agencias geográficas participantes y se presentó un método para identificación de errores gruesos en la información de gravimetría con el uso de despliegue gráfico de Google Earth.

El geoide gravimétrico para México, Centroamérica y el Caribe GGM-CA-2015 es la realización de una superficie equipotencial del campo de gravedad, parametrizado por la constante $W_{0}=62636856.0 \mathrm{~m}^{2} / \mathrm{s}^{2}$. Este modelo representa una contribución hacia la unificación mundial de los sistemas de alturas, para remover el problema de incompatibilidad entre los sistemas de alturas que existe entre zonas y países. En un primer acercamiento, se mostró una estimación de la diferencia entre dátum vertical de cuatro países en la región, que se mantiene en el rango de decímetros.

Se estima además que la exactitud absoluta de altura geoidal modelada es variable por regiones, con desviación estándar de varios decímetros, aparentemente correlacionado con la rugosidad de topografía, y diferencias máximas de 2.27 metros en contraste con el geoide derivado del EGM2008.

Para trabajo futuro se propone una profundización en la evaluación de exactitud del GGM-CA-2015, utilizando conjuntos de datos de referencia mejorados en cantidad y calidad. Se puntualiza además que existen vías de mejora para el modelado geoidal de esta región, como el desarrollo de mejores controles de calidad para 
datos gravimétricos, el aumento decidido de levantamientos sobre zonas de escaso o nulo cubrimiento y la implementación de técnicas de refinamiento al modelar el campo de gravedad en resolución similar a los $2.5^{\prime}$ que se buscó en el modelado geoidal.

La práctica de análisis gravimétrico ha permitido a representantes de ocho países participantes lo siguiente: a) incrementar la habilidad para dialogar sobre aspectos técnicos de cálculo geoidal y campo de gravedad tanto con usuarios connacionales como con instancias externas, b) abrir el acceso a software científico para realizar sus propios estudios, c) formar parte de una red de comunicación más efectiva con agencias de la región. Con esto se muestra que la estrategia de desarrollo participativo en distribución de cargas de trabajo puede darse aún en procesos complejos como el cálculo geoidal, con la desventaja de planificar sobre periodos de tiempo más extendidos que la alternativa de procesamiento centralizado, que es la más común para modelado por regiones.

\section{Agradecimientos}

Para el logro de producción del modelo geoidal, así como del presente documento se agradece el interés y las valiosas sugerencias del doctor Petr Vanicek, Profesor emérito de la Universidad de New Brunswick, del doctor Jianliang Huang, investigador del Natural Resources Canada, y del doctor Daniel Roman, investigador del National Geodetic Survey de los Estados Unidos. Se recibió apoyo para acceder a datos gravimétricos y para dar continuidad a este trabajo por parte de funcionarios directivos de los institutos geográficos participantes: Instituto Nacional de Estadística y Geografía de México (INEGI), Instituto Geográfico Nacional de Guatemala (IGN), Centro Nacional de Registros de El Salvador (CNR), Instituto de la Propiedad de Honduras (IP), Instituto Nacional de Estudios del Territorio de Nicaragua (INETER), Instituto Geográfico Nacional de Costa Rica (IGNCR), Instituto Geográfico Nacional Tommy Guardia de Panamá (IGNTG) y el Instituto Cartográfico Militar de República Dominicana (ICM).

El soporte financiero del proyecto de Geoide para México, Centroamérica y el Caribe fue proporcionado por el INEGI, por el Instituto Panamericano de Geografía e Historia (IPGH), la Secretaría de Relaciones Exteriores de México, el National Geodetic Survey de los Estados Unidos y la Universidad de New Brunswick de Canadá.

\section{Bibliografía}

Avalos-Naranjo, D.; Figueroa, C.; Medrano-Silva, W.; Ballesteros, C.; RoblesPereira, V.; Alvarez-Calderón, A.; Taveras-Polanco, L. y Meza, O. (2013). "Un diagnóstico de infraestructura geodésica en la región de Centroamérica y el Ca- 
ribe", Revista Cartográfica, núm. 89, Instituto Panamericano de Geografía e Historia, México.

Blitzkow, D.; Oliveira Cancoro, A.C.; Do Nascimento Guimarães, G.; Pacino, M.C.; Lauría, E.A.; Nunes, M., Correia e Castro Junior, C.A.; Flores, F.; Orihuela Guevara, N.; Alvarez, R. y Hernandez, J.N. (2016). "Gravity and geoid model for South America", EGU General Assembly 2016.

Ellmann, A. y Vanicek, P. (2007). "UNB application of Stokes-Helmert's approach to geoid computation", Journal of Geodynamics, vol. 43.

Huang, J.; Vanicek, P.; Pagiatakis, S.; Brink, W. (2001). "Effect of topographical density on geoid in the Canadian Rocky Mountains", Journal of Geodesy, vol. 74, pp. 805-815, Editorial Springer Verlag.

Huang, J. y Véronneau, M. (2013). "Canadian Gravimetric Model 2010”, Journal of Geodesy, vol. 87.

Hirt, C. (2013). "RTM gravity forward-modeling using topography/bathymetry data to improve high-degree global geopotential models in the coastal zone", Marine Geodesy, vol. 36, no. 2.

INEGI (2011). "Taller del Geoide para México, Centroamérica y el Caribe", Instituto Nacional de Estadística y Geografía, Aguascalientes, México, 2015.

(2013). "2do. Taller del Geoide para México, Centroamérica y el Caribe". Instituto Nacional de Estadística y Geografía, Aguascalientes, México, 2015.

(2014). "3er. Taller del Geoide para México, Centroamérica y el Caribe". Instituto Nacional de Estadística y Geografía, Aguascalientes, México, 2015.

Janak, J. and P. Vanicek, (2004). "Mean free-air anomalies in the mountains". Technical report of the Slovak University of Technology, Bratislava, 2004.

(2005). "Mean free-air gravity anomalies in the mountains", Studia Geophysica et Geodaetica, vol. 49.

(2011). "Numerical evaluation of mean values of topographical effects", Journal of Geodetic Science, vol. 1(2).

Kingdon, R. and Vanicek, P. (2011). "Poisson downward continuation solution by the Jacoby method", Journal of Geodetic Science, Editorial De Gruyter.

Martinec, Z.; Vanicek, P.; Mainville, A. and Véronneau, M. (1996). "Evaluation of topographical effects in precise geoid computation from densely sampled heights", Journal of Geodesy, vol. 70, pp. 746-754, Editorial Springer Verlag.

Mayer-Gürr, T.; A. Kvas; B. Klinger; D. Rieser; N. Zehentner; R. Pail; T. Gruber; T. Fecher; M. Rexer; W.D. Schuh; J. Kusche; J.M. Brockmann; I. Loth; S. Müller; A. Eicker; J. Schall; O. Baur; E. Höck; S. Krauss; A. Jaggi; U. Meyer; L. Prange and A. Maier (2015). "The combined satellite gravity field model GOCO05s”. Presentación de la EGU 2015, Vienna, April. 
Pavlis, N.K.; Holmes, S.A.; Kenyon, S.C. and Factor, J.K. (2012). "The development and evaluation of the Earth Gravitational Model 2008 (EGM2008)". Journal of Geophysical Research, Solid Earth, vol. 117.

Sánchez, L.; R. Čunderlík; N. Dayoub; K. Mikula; Z. Minarechová; Z. Šíma; V. Vatrt and M. Vojtíšková, 2016. "A conventional value for the geoid reference potential Wo", Journal of Geodesy, 90(9).

Sun, W. and Vanicek, P. (1998). "On some problems of the downward continuation of the 5' x 5' mean Helmert gravity disturbance". Journal of Geodesy, vol. 72, Editorial Springer Verlag.

UN-GGIM, 2016. "Road Map for the Global Geodetic Reference Frame for Sustainable Development", Reporte E/C.20/2016/4, Sexta sesión, Nueva York, agosto, 2016.

Vanicek, P. and Sjöberg, L.E. (1991). "Reformulation of Stokes's theory for higher $\tan$ second-degree reference field and modification of integration kernels", Journal of Geophysical Research, vol. 96, pp. 6529-6539.

Vanicek, P.; R. Kingdon; M. Kuhn; A. Ellmann; W. Featherstone; M.C. Santos; Z. Martinec; C. Hirt; D. Avalos (2013). "Testing Stokes-Helmert geoid model computation on a synthetic gravity field: experiences and shortcomings", Studia Geophysica et Geodaetica, 57(3). 